\title{
Fractional dynamic system simulating the growth of microbe
}

\author{
Samir B. Hadid ${ }^{1,2^{*}}$ (D) and Rabha W. Ibrahim³
}

"Correspondence:

s.hadid@ajman.ac.ae

${ }^{1}$ Nonlinear Dynamics Research Center (NDRC), Ajman University, Ajman, UAE

${ }^{2}$ Department of Mathematics and Sciences, College of Humanities and Sciences, Ajman University, 346 Ajman, UAE

Full list of author information is available at the end of the article

\begin{abstract}
There are different approaches that indicate the dynamic of the growth of microbe. In this research, we simulate the growth by utilizing the concept of fractional calculus. We investigate a fractional system of integro-differential equations, which covers the subtleties of the diffusion between infected and asymptomatic cases. The suggested system is applicable to distinguish the presentation of growth level of the infection and to approve if its mechanism is positively active. An optimal solution under simulation mapping assets is considered. The estimated numerical solution is indicated by employing the fractional Tutte polynomials. Our methodology is based on the Atangana-Baleanu calculus (ABC). We assess the recommended system by utilizing real data.
\end{abstract}

Keywords: Fractional calculus; Fractional differential operator; Fractional equation

\section{Introduction}

Integro-differential dynamic system of equations simulates various states from science and engineering corresponding to the analysis, control, and optimization studies. The main model in this direction is the Wilson-Cowan system, which designs the dynamics of connections between populations of very inhibitory system in cells or neurons. It was developed by Hugh R. Wilson and Jack D. Cowan [1]. The system and its generalizations have been extensively utilized in forming neuronal or cell populations [2]. The system is significant traditionally because it utilizes phase plane approaches and mathematical solutions to designate the reactions of neuronal populations to motivations. The general system involves simple integro-differential equations, therefore, limit cycle performance (neural fluctuations) and stimulus-dependent suggested reactions are expected. The key results contain the solvability of multiple stable situations and hysteresis in the population's reaction.

Coronavirus (COVID-19) has been an infectious virus molded by a recently exposed coronavirus. It has been recorded by the World Health Organization (WHO), it is a pandemic. The first WHO warning of dyed-in-the-wool cases of COVID-19 indicated on January 2020 with 282 cases $($ see $[3,4])$. There is an increasing number of research works that develop the growth of the COVID-19 infection by using an ordinary dynamic system [5] and fractal-fractional dynamic system [6]. Utilizing the recent information from Euro-

(c) The Author(s) 2021. This article is licensed under a Creative Commons Attribution 4.0 International License, which permits use, sharing, adaptation, distribution and reproduction in any medium or format, as long as you give appropriate credit to the original author(s) and the source, provide a link to the Creative Commons licence, and indicate if changes were made. The images or other third party material in this article are included in the article's Creative Commons licence, unless indicated otherwise in a credit line to the material. If material is not included in the article's Creative Commons licence and your intended use is not permitted by statutory regulation or exceeds the permitted use, you will need to obtain permission directly from the copyright holder. To view a copy of this licence, visit http://creativecommons.org/licenses/by/4.0/. 
pean and African countries, Atangana and Araz presented numerous statistical analyses [7, 8]. Musa et al. [9] introduced a nonlinear 4D-system of ordinary differential equations describing COVID-19. Atangana [10] formulated a numerical design using the Newton polynomial. Other strategies can be located in efforts by Memon et al. [11]. Newly, numerous mathematical simulations have been indicated to realize the coronavirus infection. Supreme of these representations are based on classical integer-order derivative or classical fractional differential operators, which cannot get the vanishing memory and boundary performance found in numerous biological phenomena. Consequently, we investigate the coronavirus disease in this study by discovering the dynamics of COVID-19 infection utilizing the fractional Caputo derivative.

The terminal coronavirus continues to blow out across the globe, and mathematical models can be utilized to display suspected, recovered, and deceased coronavirus patients, as well as how many persons have been tested or even vaccinated. Consequently, mathematical and statistical solutions of the infected human beings overall can decrease the risk of future COVID-19 spread. In this study, we aim to generalize the Wilson-Cowan system (WCS) utilizing the concept of fractional calculus to study the growth of COVID19 population. This investigation includes a dynamic term, which is the exponential law to discover and realize the graph of the growth. The solvability of the system is indicated by using the optimal point theorem of simulation function. Other behaviors are indicated such as the approximated solvability using the fractional Tutte polynomials.

\section{Preparations}

This section deals with some concepts and the properties of these concepts.

\subsection{ABC-definition}

In recent decades, numerous physical issues have been exposed using the fractional calculus. The essential explanations for employing fractional calculus are that various measures, structures, and inequities display capability to remember the past or nonlocal possessions. The basic outlook and appearances of fractional calculus and fractional differential equations are recognized in various reviews. Most researches focus on the derivatives, which include kernels. For instance, the main difference between the Caputo operator, the Caputo-Fabrizio operator [12], and others is that the Caputo operator is communicated by giving a power law, the Caputo-Fabrizio operator is adapted by utilizing an exponential growth act. The Atangana-Baleanu operator is presented by signifying the extended Mittag-Leffler function [13].

Definition 2.1 Let $\Lambda^{\mu}, \mu \in(0,1)$ be the Atangana-Baleanu operator of order $\mu$ of a function $\chi$ fulfilling

$$
\Lambda^{\mu} \chi(t)=\frac{B(\mu)}{1-\mu} \int_{0}^{t} \chi^{\prime}(\tau) \Xi_{\mu}\left(\frac{-\mu}{1-\mu}(t-\tau)^{\mu}\right) d \tau, \quad t \in[0, \infty),
$$

where $B(\mu)$ indicates a normalization function, $\Xi$ represents the Mittag-Leffler function. Corresponding to $\Lambda^{\mu}$, the $\mathrm{ABC}$ integral is realized by

$$
J^{\mu} \chi(t)=\frac{(1-\mu)}{B(\mu)} \chi(t)+\frac{\mu}{B(\mu) \Gamma(\mu)} \int_{0}^{t} \chi(\tau)(t-\tau)^{\mu-1} d \tau .
$$


Example 2.1 Consider the function $\chi(t)=t^{m}$, then the ABC integral becomes

$$
J^{\mu} t^{m}=\frac{(1-\mu)}{B(\mu)} t^{m}+\frac{\mu \Gamma(m+1)}{B(\mu) \Gamma(m+1+\mu)} t^{m+\mu} .
$$

In our study, since we focus on the approximated solutions, we assume that $B(\mu) \rightarrow 1$ for all $\mu \in(0,1)$. Applications of this calculus in COVID-19 can be located in [14-16].

\subsection{Approximate point theorem}

We deal with the resulting notion of cyclic $\complement$-condensing operator. Let $\partial$ be a measure of noncompactness on a Banach space $\mathbb{X}$ and $A, B$ be nonempty and convex subsets of $\mathbb{X}$ (see [17]).

Definition 2.2 A function $f: \mathbb{R}_{+} \times \mathbb{R}_{+} \rightarrow \mathbb{R}$ is called simulation if and only if $f(0,0)=0$, $f\left(x_{1}, x_{2}\right)<x_{2}-x_{1}$, where $x_{2}, x_{1}>0$ and $\lim _{i \rightarrow \infty} \sup f\left(x_{i}, y_{i}\right)<0$ for $x_{i}<y_{i}$ and $\lim _{i \rightarrow \infty} x_{i}=$ $\lim _{i \rightarrow \infty} y_{i}=0$.

Definition 2.3 Let $Y: A \cup B \rightarrow A \cup B$ be an operator. It is $f$-cyclic condensing (CC) if, for every nonempty, bounded, convex, and closed subset $\left(A_{1}, B_{1}\right)$,

$$
\left.f\left[ð\left(Y\left(A_{1}\right) \cup Y\left(B_{1}\right)\right]\right), \partial\left(A_{1} \cup B_{1}\right)\right] \geq 0, \quad\left(A_{1}, B_{1}\right) \subset(A, B) .
$$

Lemma 2.2 A relatively nonexpansive cyclic $f$-condensing mapping $Y: A \cup B \rightarrow A \cup B$ recognizes a best proximity point.

\section{Results}

We let $\mathbb{N}(t)$ be the accumulation number of infected people, which represents the sum of the number of the standard infected persons $\chi(t)$ and that of the asymptomatic transmission ones $\Upsilon(t): \mathbb{N}(t)=\chi(t)+\Upsilon(t)$. Take into account that $\chi(t)$ includes people who have previously been diseased. Therefore, there are rate functions joining $\chi$ and $\Upsilon$. The following integro-differential system indicates the generalization of WCS. We suggest the generalization by using the $\mathrm{ABC}$ formula of fractional calculus as follows:

$$
\begin{aligned}
& \Lambda^{\mu} \chi(\varsigma)=\Psi_{1}\left(\varsigma, \chi(\varsigma), \int_{\tau_{1}}^{\tau_{1}+\tau_{2}} \phi_{1}(\varsigma, \tau, \chi(\tau)) d \tau, \int_{\tau_{1}}^{\varsigma} \psi_{1}(\varsigma, \tau, \chi(\tau)) d \tau\right), \\
& \chi\left(\tau_{1}\right)=\chi_{1}, \\
& \Lambda^{\mu} \Upsilon(\varsigma)=\Psi_{2}\left(\varsigma, \Upsilon(\varsigma), \int_{\tau_{1}}^{\tau_{1}+\tau_{2}} \phi_{2}(\varsigma, \tau, \Upsilon(\tau)) d \tau, \int_{\tau_{1}}^{\varsigma} \psi_{2}(\varsigma, \tau, \Upsilon(\tau)) d \tau\right), \\
& \Upsilon\left(\tau_{1}\right)=\Upsilon_{1},
\end{aligned}
$$

where the variables are defined in different intervals: $\Pi=\left[\tau_{1}-\tau_{2}, \tau_{1}+\tau_{2}\right], \Pi_{\chi}=\left[\chi_{1}-\right.$ $\left.\epsilon, \chi_{1}+\epsilon\right], \Pi_{\Upsilon}=\left[\Upsilon_{1}-\epsilon, \Upsilon_{1}+\epsilon\right]$, and $\Pi_{\epsilon}=\left[\tau_{1}-\epsilon, \tau_{1}+\epsilon\right]$.

To study the solvability of system (1), we formulate the following assumptions:

(A1) All the functions are continuous in $\mathbb{R}$ such that $\phi_{1}: \Pi \times \Pi \times \Pi_{\chi} \rightarrow \mathbb{R}$, $\phi_{2}: \Pi \times \Pi \times \Pi_{\Upsilon} \rightarrow \mathbb{R}, \Psi_{1}: \Pi_{\epsilon} \times \Pi_{\chi} \times \Pi_{\chi} \times \Pi_{\chi} \rightarrow \mathbb{R}$,

$\Psi_{2}: \Pi_{\epsilon} \times \Pi_{\Upsilon} \times \Pi_{\Upsilon} \times \Pi_{\Upsilon} \rightarrow \mathbb{R}$ and $\chi, \Upsilon$ are inside the nonempty, bounded, closed, and convex sets $\mathrm{T}_{1} \subset C\left(\Pi_{\epsilon}, \mathbb{R}\right)$ and $\mathrm{T}_{2} \subset C\left(\Pi_{\epsilon}, \mathbb{R}\right)$ respectively. 
(A2) For a sup.norm, we suppose that $\left\|\chi_{1}-\Upsilon_{1}\right\| \leq \epsilon\|\chi-\Upsilon\|, 0<\epsilon \leq 1$, so that $\operatorname{dis}\left(T_{1}, T_{2}\right)=\left\|\chi_{1}-\Upsilon_{1}\right\|$. In addition, for all $\chi \in T_{1}$ and $\Upsilon \in T_{2}$, we suppose that there occurs a positive constant $\rho>0$ fulfilling

$$
\left\|\Psi_{1}-\Psi_{2}\right\| \leq \rho\left(\|\chi-\Upsilon\|-\left\|\chi_{1}-\Upsilon_{1}\right\|\right)
$$

(A3) For any $\Pi_{\chi}, \Pi_{\Upsilon}$, there exists a positive function $\mathbb{k}: \mathbb{R}^{+} \rightarrow \mathbb{R}^{+}$which is upper semi-continuous and achieves $\mathbb{k}(l)<\imath$ and

$$
\begin{aligned}
& \partial\left(\Upsilon_{1}\left(\Pi_{\epsilon} \times \Pi_{\chi} \times \Pi_{\chi} \times \Pi_{\chi}\right) \cup \Psi_{2}\left(\Pi_{\epsilon} \times \Pi_{\Upsilon} \times \Pi_{\Upsilon} \times \Pi_{\Upsilon}\right)\right) \\
& \quad<\frac{\mathbb{k}\left(\partial\left(\Pi_{\chi} \cup \Pi_{\Upsilon}\right)\right)}{\left[(1-\mu)+\frac{\bar{\epsilon}_{\mu}}{\Gamma(\mu)}\right]}, \quad \bar{\epsilon}_{\mu}>0 .
\end{aligned}
$$

Here, we introduce our theorem for the solvability of system (1). We define an operator $\mathbb{Q}: \mathrm{T}_{1} \cup \mathrm{T}_{2} \rightarrow C\left(\Pi_{\epsilon}, \mathbb{R}\right)$ as follows:

$$
\mathbb{Q}(\varsigma):= \begin{cases}\Upsilon_{1}+(1-\mu) \Psi_{1}+\frac{\mu}{\Gamma(\mu)} \int_{\tau_{1}}^{\varsigma} \Psi_{1}(\eta)(\varsigma-\eta)^{\mu-1} d \eta & \text { if } \chi \in \mathrm{T}_{1}, \\ \chi_{1}+(1-\mu) \Psi_{2}+\frac{\mu}{\Gamma(\mu)} \int_{\tau_{2}}^{\varsigma} \Psi_{2}(\eta)(\varsigma-\eta)^{\mu-1} d \eta & \text { if } \Upsilon \in \mathrm{T}_{2}\end{cases}
$$

Theorem 3.1 Consider system (1) satisfying hypotheses (A1)-(A3). Then it has an optimal solution in $C\left(\Pi_{\epsilon}, \mathbb{R}\right)$, whenever

$$
\begin{gathered}
\rho<\frac{\Gamma(\mu)}{\Gamma(\mu)+(1-\mu)+\bar{\epsilon}_{\mu}}, \\
\left(\rho>0,0<\mu<1, \bar{\epsilon}_{\mu}>0\right) .
\end{gathered}
$$

Proof Consider the operator $\mathbb{Q}$ and $B(\mu) \rightarrow 1$. We aim to show that $\mathbb{O}$ is a cyclic operator. Let $\chi \in \mathrm{T}_{1}$, then we get

$$
\begin{aligned}
\left\|(\mathbb{Q} \chi)-\Upsilon_{1}\right\| & =\left\|(1-\mu) \Psi_{1}+\frac{\mu}{\Gamma(\mu)} \int_{\tau_{1}}^{\varsigma} \Psi_{1}(\eta)(\varsigma-\eta)^{\mu-1} d \eta\right\| \\
& \leq(1-\mu)\left\|\Psi_{1}\right\|+\frac{\mu}{\Gamma(\mu)} \int_{\tau_{1}}^{\varsigma}\left\|\Psi_{1}(\eta)(\varsigma-\eta)^{\mu-1}\right\| d \eta \\
& \leq(1-\mu)\left\|\Psi_{1}\right\|+\frac{\mu}{\Gamma(\mu)}\left\|\Psi_{1}\right\| \int_{\tau_{1}}^{\varsigma}(\varsigma-\eta)^{\mu-1} d \eta \\
& \leq\left\|\Psi_{1}\right\|\left(1+\frac{\left(\varsigma-\tau_{1}\right)^{\mu}}{\Gamma(\mu)}\right) \\
& :=\left\|\Psi_{1}\right\|\left(1+\frac{\epsilon_{1}^{\mu}}{\Gamma(\mu)}\right) \\
& :=S_{1}\left({ }_{1} \epsilon_{\mu}\right), \quad{ }_{1} \epsilon_{\mu}:=1+\frac{\epsilon_{1}^{\mu}}{\Gamma(\mu)}
\end{aligned}
$$

where $S_{1}:=\sup \left(\Psi_{1}\right)=\left\|\Psi_{1}\right\|$. By letting $\left({ }_{1} \epsilon_{\mu}\right)<\frac{\bar{\epsilon}_{\mu}}{\max \left\{S_{1}, S_{2}\right\}}$, where $\bar{\epsilon}_{\mu}:=\max \left\{{ }_{1} \epsilon_{\mu}, 2 \epsilon_{\mu}\right\}$ and $S_{2}:=\sup \left(\Psi_{2}\right)$, we have

$$
\left\|(\mathbb{Q} \chi)-\Upsilon_{1}\right\|<\bar{\epsilon}_{\mu}, \quad \forall \chi \in \mathrm{T}_{1} .
$$


Thus, $\mathbb{Q} \chi \in \mathrm{T}_{2}$. In the same manner, we conform that, for $\Upsilon \in \mathrm{T}_{2}$, this indicates that

$$
\left\|\mathbb{Q} \Upsilon-\chi_{1}\right\|<\bar{\epsilon}_{\mu}
$$

and hence $\mathbb{Q} y \in \mathrm{T}_{1}$. We conclude that $\mathbb{Q}$ is cyclic. The above conclusion shows that the set $\mathbb{Q}\left(\mathrm{T}_{1}\right)$ is bounded in $\mathrm{T}_{2}$, and the set $\mathbb{Q}\left(\mathrm{T}_{2}\right)$ is bounded in $\mathrm{T}_{1}$.

Recall that $\phi \in \mathrm{T}_{1} \cup_{\mathrm{T}_{2}}$ indicates an optimum outcome of system (1) if and only if $\operatorname{dist}\left(\mathrm{T}_{1} \cup\right.$ $\left.T_{2}\right)=\|\phi-\mathbb{Q} \phi\|$. Therefore, we have to prove this fact. Next, we aim to prove that $\mathbb{Q}\left(\mathrm{T}_{1}\right)$ is equicontinuous in $\mathrm{T}_{2}$. For $\varsigma$ and $\varsigma^{\prime}$, we have

$$
\begin{aligned}
& \left\|\mathbb{Q} \chi(\varsigma)-\mathbb{O} \chi\left(\varsigma^{\prime}\right)\right\| \\
& \quad=\frac{\mu}{\Gamma(\mu)}\left\|\int_{\tau_{1}}^{\varsigma} \Psi_{1}(\eta)(\varsigma-\eta)^{\mu-1} d \eta-\int_{\tau_{1}}^{\varsigma^{\prime}} \Psi_{1}(\eta)\left(\varsigma^{\prime}-\eta\right)^{\mu-1} d \eta\right\| \\
& \quad \leq \frac{\mu}{\Gamma(\mu)}\left|\int_{\varsigma}^{\varsigma^{\prime}}\left\|\Psi_{1}(\eta)\right\|\left(\varsigma-\varsigma^{\prime}\right)^{\mu-1} d \eta\right| \\
& \quad \leq \frac{1}{\Gamma(\mu)} S_{1}\left|\varsigma-\varsigma^{\prime}\right|^{\mu} \\
& \quad \leq \bar{\epsilon}_{\mu} S_{1}
\end{aligned}
$$

which implies that $\mathbb{Q}\left(T_{1}\right)$ is equicontinuous in $T_{2}$. In a similar manner, we confirm that $\mathbb{Q}\left(\mathrm{T}_{2}\right)$ is equicontinuous in $\mathrm{T}_{1}$. As a consequence and via the Arzela-Ascoli theorem, we point that the pair $\left(\mathrm{T}_{1}, \mathrm{~T}_{2}\right)$ is relatively compact. Now, we have to show that $\mathbb{Q}$ is relatively nonexpansive.

For $(\chi, \Upsilon) \in\left(\mathrm{T}_{1}, \mathrm{~T}_{2}\right)$, we inform that

$$
\begin{aligned}
\|\mathbb{Q} \chi(\varsigma)-\mathbb{Q} \Upsilon(\varsigma)\| & \| \Upsilon_{1}+(1-\mu) \Psi_{1}+\frac{\mu}{\Gamma(\mu)} \int_{\tau_{1}}^{\varsigma} \Psi_{1}(\varsigma-\eta)^{\mu-1}(\eta) d \eta \\
& \quad-\chi_{1}-(1-\mu) \Psi_{2}-\frac{\mu}{\Gamma(\mu)} \int_{\tau_{1}}^{\varsigma} \Psi_{2}(\eta)(\varsigma-\eta)^{\mu-1} d \eta \| \\
\leq & \left\|\chi_{1}-\Upsilon_{1}\right\|+(1-\mu)\left\|\Psi_{1}(\varsigma)-\Psi_{2}(\varsigma)\right\| \\
& +\frac{\mu}{\Gamma(\mu)}\left|\int_{\tau_{1}}^{\varsigma}\left\|\Upsilon_{1}(\eta)-\Upsilon_{2}(\eta)\right\|(\varsigma-\eta)^{\mu-1} d \eta\right| \\
\leq & \epsilon\|\chi-\Upsilon\|+(1-\mu) \rho\left(\|\chi-\Upsilon\|-\left\|\chi_{1}-\Upsilon_{1}\right\|\right)+\frac{\rho \bar{\epsilon}_{\mu}}{\Gamma(\mu)}\left(\|\chi-\Upsilon\|-\left\|\chi_{1}-\Upsilon_{1}\right\|\right) \\
\leq & \epsilon\|\chi-\Upsilon\|+(1-\mu) \rho\left(\|\chi-\Upsilon\|-\left\|\chi_{1}-\Upsilon_{1}\right\|\right)+\frac{\rho \bar{\epsilon}_{\mu}}{\Gamma(\mu)}\left(\|\chi-\Upsilon\|-\left\|\chi_{1}-\Upsilon_{1}\right\|\right) \\
= & {\left[\epsilon+(1-\mu) \rho+\frac{\rho \bar{\epsilon}_{\mu}}{\Gamma(\mu)}\right]\|\chi-\Upsilon\|-\left[(1-\mu) \rho+\frac{\rho \bar{\epsilon}_{\mu}}{\Gamma(\mu)}\right]\left\|\chi_{1}-\Upsilon_{1}\right\| . }
\end{aligned}
$$


But $\epsilon$ is an arbitrary constant, thus when $\epsilon \rightarrow 0$, we have the inequality

$$
\begin{aligned}
\|\mathbb{Q} \chi(\varsigma)-\mathbb{Q} \Upsilon(\varsigma)\| & \leq\left[(1-\mu) \rho+\frac{\rho \bar{\epsilon}_{\mu}}{\Gamma(\mu)}\right](\|\chi-\Upsilon\|) \\
& <\|\chi-\Upsilon\| .
\end{aligned}
$$

This indicates that $\mathbb{Q}$ is relatively nonexpansive.

We proceed to show that $\mathbb{Q}$ is $f$-condensing. Assume that $\left(\Pi_{\chi}, \Pi_{\Upsilon}\right) \subseteq\left(\mathrm{T}_{1}, \mathrm{~T}_{2}\right)$ is a nonempty, bounded, closed, and convex set such that

$$
\begin{aligned}
& \partial\left(\mathbb{Q}\left(\Pi_{\chi}\right) \cup \mathbb{Q}\left(\Pi_{\Upsilon}\right)\right) \\
& =\max \left\{\left(\partial \mathbb{Q}\left(\Pi_{\chi}\right), \partial \mathbb{Q}\left(\Pi_{\Upsilon}\right)\right)\right\} \\
& =\max \left(\sup _{\chi}\left\{\partial \mathbb{Q} \chi, \chi \in \Pi_{\chi}\right\}, \sup _{\Upsilon}\left\{\partial \mathbb{Q} \Upsilon, \in \Pi_{\Upsilon}\right\}\right) \\
& =\max \left(\sup _{\chi}\left\{\partial\left(\Upsilon_{1}+(1-\mu) \overline{\operatorname{conv}} \Psi_{1}+\frac{\bar{\epsilon}_{\mu}}{\Gamma(\mu)} \overline{\operatorname{conv}} \Psi_{1}\right)\right\},\right. \\
& \left.\sup _{\Upsilon}\left\{\partial\left(\chi_{1}+(1-\mu) \operatorname{con} \Psi_{2}+\frac{\bar{\epsilon}_{\mu}}{\Gamma(\mu)} \overline{\operatorname{conv}} \Psi_{2}\right)\right\}\right) \\
& \leq \max \left(\operatorname { s u p } _ { \chi } \left\{\partial \left(\Upsilon_{1}+(1-\mu) \overline{\operatorname{conv}} \Psi_{1}\left(\Pi_{\epsilon} \times \Pi_{\chi} \times \Pi_{\chi} \times \Pi_{\chi}\right)\right.\right.\right. \\
& \left.\left.+\frac{\bar{\epsilon}_{\mu}}{\Gamma(\mu)} \overline{\operatorname{conv}} \Psi_{1}\left(\Pi_{\epsilon} \times \Pi_{\chi} \times \Pi_{\chi} \times \Pi_{\chi}\right)\right)\right\}, \\
& \sup _{\Upsilon}\left\{\partial \left(\chi_{1}+(1-\mu) \overline{\operatorname{conv}} \Psi_{2}\left(\Pi_{\epsilon} \times \Pi_{\Upsilon} \times \Pi_{\Upsilon} \times \Pi_{\Upsilon}\right)\right.\right. \\
& \left.\left.\left.+\frac{\bar{\epsilon}_{\mu}}{\Gamma(\mu)} \overline{\operatorname{conv}} \Psi_{2}\left(\Pi_{\epsilon} \times \Pi_{\Upsilon} \times \Pi_{\Upsilon} \times \Pi_{\Upsilon}\right)\right)\right\}\right) \\
& \leq\left[(1-\mu)+\frac{\bar{\epsilon}_{\mu}}{\Gamma(\mu)}\right] \max \left(\left\{\partial\left(\Upsilon_{1}\left(\Pi_{\epsilon} \times \Pi_{\chi} \times \Pi_{\chi} \times \Pi_{\chi}\right)\right)\right\},\right. \\
& \left.\left\{\chi\left(\Upsilon_{2}\left(I_{\epsilon} \times \Pi_{\Upsilon} \times \Pi_{\Upsilon} \times \Pi_{\Upsilon}\right)\right)\right\}\right) \\
& \leq\left[(1-\mu)+\frac{\bar{\epsilon}_{\mu}}{\Gamma(\mu)}\right] \partial\left(\Upsilon_{1}\left(\Pi_{\epsilon} \times \Pi_{\chi} \times \Pi_{\chi} \times \Pi_{\chi}\right) \cup \Upsilon_{2}\left(\Pi_{\epsilon} \times \Pi_{\Upsilon} \times \Pi_{\Upsilon} \times \Pi_{\Upsilon}\right)\right) \\
& <\left[(1-\mu)+\frac{\bar{\epsilon}_{\mu}}{\Gamma(\mu)}\right] \frac{\mathbb{k}\left(ð\left(\Pi_{\chi} \cup \Pi_{\Upsilon}\right)\right)}{\left[(1-\mu)+\frac{\bar{\epsilon}_{\mu}}{\Gamma(\mu)}\right]} \\
& =\mathbb{k}\left(ð\left(\Pi_{\chi} \cup \Pi_{\Upsilon}\right)\right) \text {. }
\end{aligned}
$$

Thus, we obtain

$$
\mathbb{k}\left(ð\left(\Pi_{\chi} \cup \Pi_{\curlyvee}\right)\right)-\partial\left(\mathbb{Q}\left(I_{x}\right) \cup \mathbb{Q}\left(\Pi_{\Upsilon}\right)\right) \geq 0 .
$$

By putting $f(\tau, \varsigma):=\mathbb{k}(\varsigma)-\tau$, then we arrive at

$$
f\left(ð\left(\mathbb{Q}\left(\Pi_{\chi}\right) \cup \mathbb{Q}\left(\Pi_{\curlyvee}\right)\right), \mathbb{k}\left(ð\left(\Pi_{\chi} \cup \Pi_{\Upsilon}\right)\right)\right) \geq 0 .
$$


Hence, the necessary requirements of Lemma 2.2 are achieved. Thus, the operator $\mathbb{Q}$ has the best proximity point and thus system (1) has an optimal solution.

This completes the proof.

\subsection{Numerical structures}

In this subsection, we introduce some numerical systems pointing to utilize Theorem 3.1. The chief assumption in Theorem 3.1 is $\rho<\frac{\Gamma(\mu)}{\Gamma(\mu)+(1-\mu)+\bar{\epsilon}_{\mu}}$. This inequality is very informal to fulfill likening with other existence theorems attaining (A1-A3). Theorem 3.1 designates that the system attractive formula (1) admits an optimal solution. This kind of result is very significant in dynamic and control systems. By this result, one can investigate the stability, oscillatory solution, and other performances of the resolution.

Example 3.2

$$
\begin{array}{ll}
\Lambda^{0.9} \chi(\varsigma)=\chi\left(v_{1}-v_{2} \Upsilon\right), & \chi(0)=\chi_{0}, \\
\Lambda^{0.9} \Upsilon(\varsigma)=\Upsilon\left(v_{3} \chi-v_{4}\right), & \Upsilon(0)=\Upsilon_{0} .
\end{array}
$$

By using Mathematica 11.2, the solution is realized by the integral

$$
\begin{aligned}
& \int_{\tau_{0}=0}^{\chi[\varsigma] 1 / \tau} \frac{1}{\left(1+W\left(-\left(v_{2}\right) /\left(v_{1}\right) \exp \left(\left(v_{3} \tau-c\right) / v_{1}\right) \tau^{\left.\left.-v_{4} v_{1}\right)\right)}\right.\right.} d \tau \approx v_{1} \varsigma+c, \\
& \Upsilon[\varsigma]=\left(-v_{1} / v_{2}\right) \times W\left(\left(-v_{2} / v_{1}\right) \exp \left(\left(v_{3} \chi[\varsigma]-c\right) / v_{1}\right) \chi[\varsigma]^{-v_{4} / v_{1}}\right),
\end{aligned}
$$

where $c$ is a constant and $W$ represents the product log function. As an application of Theorem 3.1, we assume that $\left(\chi_{0}, \Upsilon_{0}\right)=(1,1)$ and $\rho=\left(v_{2} v_{3}-v_{1} v_{4}\right)$, where

$$
\rho<\frac{\Gamma(\mu)}{\Gamma(0.9)+(1-0.9)+\bar{\epsilon}_{0.9}}=\frac{1.06}{3.10}=0.341 .
$$

For instance, $\left(v_{1}, v_{2}, v_{3}, v_{4}\right)=(2,1,0.4,0.1)$, we attain $\rho=0.2<0.341$; thus, by Theorem 3.1, system (3) admits an optimal solution converging to a limit cycle. In another case, suppose that $\left(v_{1}, v_{2}, v_{3}, v_{4}\right)=(1,1,0.9,0.8)$, then $\rho=0.1<0.341$. This implies that system (3) admits optimal solution converging to a limit cycle. Similarly, for $\left(v_{1}, v_{2}, v_{3}, v_{4}\right)=(1,1,1,0.8) \Rightarrow$ $\rho=0.2<0.341$ and $\left(v_{1}, v_{2}, v_{3}, v_{4}\right)=(1,0.9,1,0.8) \Rightarrow \rho=0.1<0.341$. Figure 1 represents various cases considering the value of $\rho$.

Example 3.3 Consider the following system:

$$
\begin{aligned}
& \Lambda^{0.5} \chi(\varsigma)=\Upsilon, \quad \chi(0)=\chi_{0} \\
& \Lambda^{0.5} \Upsilon(\varsigma)=-\chi+\rho \Upsilon, \quad \Upsilon(0)=\Upsilon_{0},
\end{aligned}
$$

where the value of $\rho$ achieves

$$
\rho<\frac{\Gamma(0.5)}{\Gamma(0.5)+(1-0.5)+\bar{\epsilon}_{0.5}}=\frac{1.77}{3.83}=0.462 .
$$



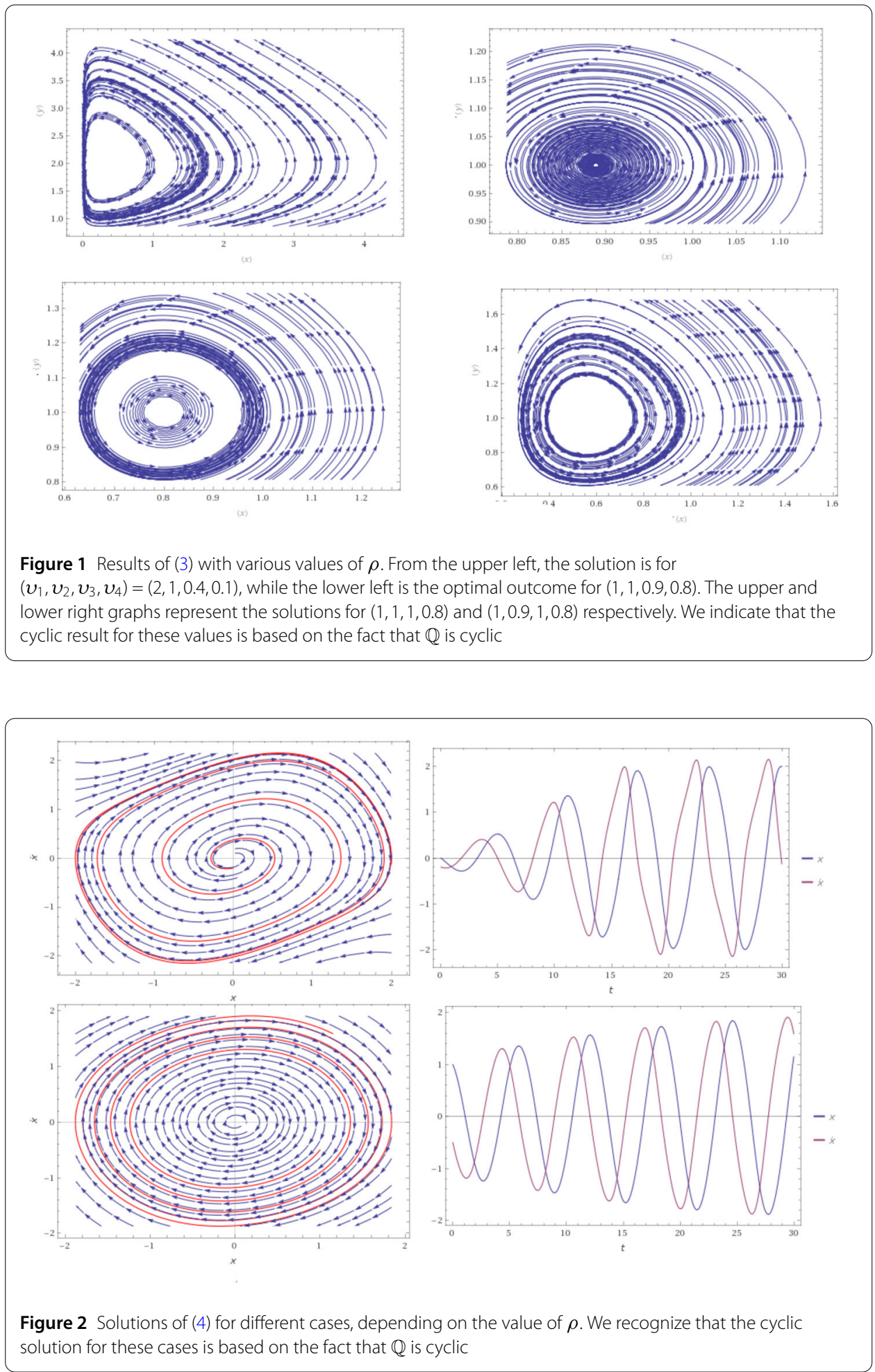

For instance, when $\rho=0.4$, we have an optimal solution with the initial condition $\left(\chi_{0}, \Upsilon_{0}\right)=$ $(0,0)$. Furthermore, it is unstable cyclic because it indicates a portrait unstable limit cycle (see Fig. 2, the upper graphs). When $\rho=0.1$, the system admits an optimal solution with a portrait unstable limit cycle (see Fig. 2, the lower graphs). 


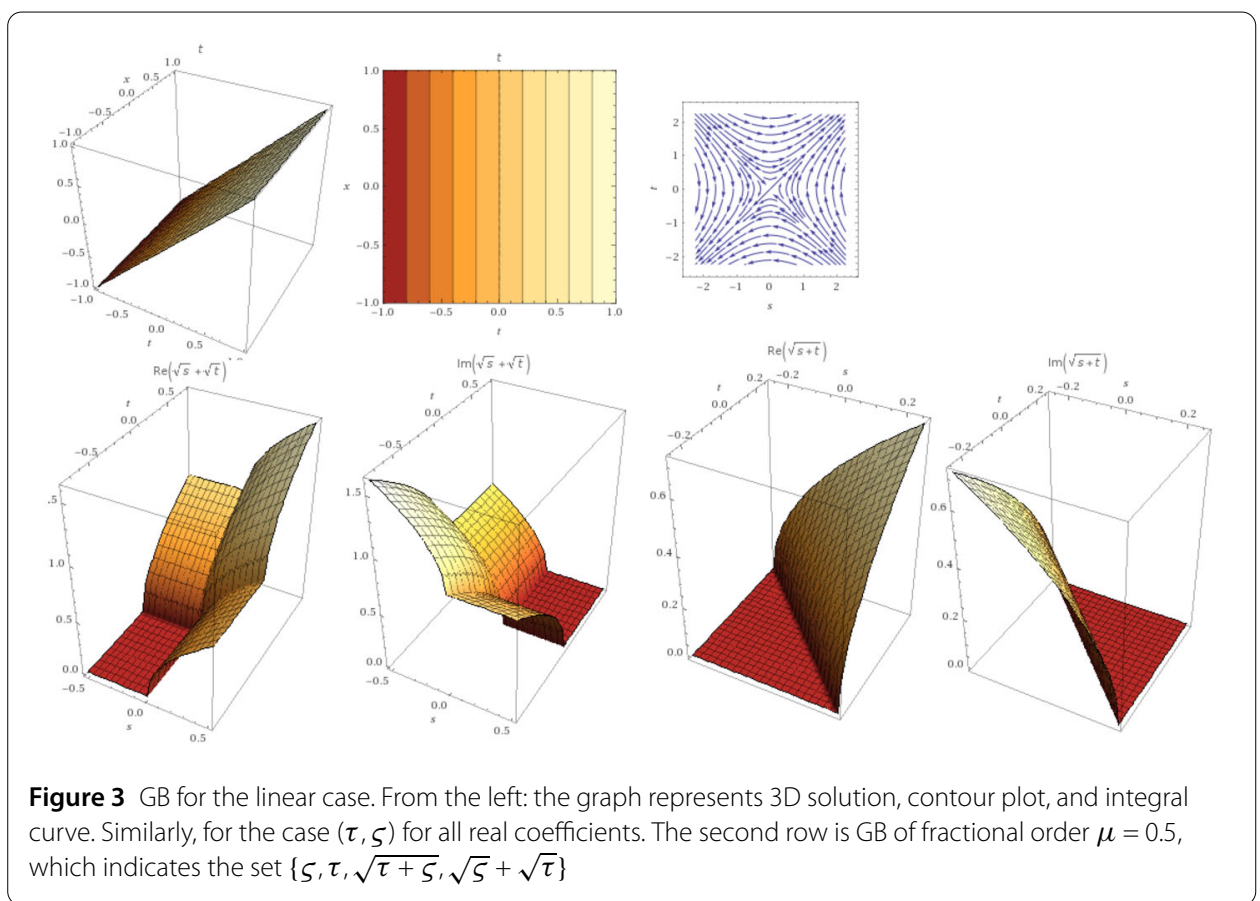

Remark 3.1 The upper value of $\rho$ plays an important role in describing the behavior of the optimal solution. It represents that when the upper value of $\rho$ is equal to 0.33 , then we have a stable solution (see Example 3.2). If the value is greater than 0.33, we have an unstable optimal solution (see Example 3.3). We shall take into account this fact when we establish the connections of Tutte polynomials in the next section.

\subsection{Approximated solution}

In this section, we aim to use a type of polynomial to approximate the solution of (1). In this place, we suggest to use the Tutte polynomial because the formality of this polynomial involves two variables as follows (see [18]):

$$
T_{m}(\varsigma, \tau)=\sum_{0 \leq i \leq m, 0<j<m} \omega(i, j) \varsigma^{i} \tau^{j} .
$$

By using the construction of Example 2.1, we have the fractional Tutte polynomial as follows:

$$
\begin{aligned}
T_{m}^{\mu}(\varsigma, \tau):= & J^{\mu} T_{m}(\varsigma, \tau) \\
= & (1-\mu) T_{m}(\varsigma, \tau)+\frac{\mu}{\Gamma(\mu)} \int_{0}^{\varsigma} T_{m}(\tau, \tau)(\varsigma-\tau)^{\mu-1} d \tau \\
= & (1-\mu) \sum_{0 \leq i \leq m, 0<j<m} \omega(i, j) \varsigma^{i} \tau^{j} \\
& +\frac{\mu}{\Gamma(\mu)} \int_{0}^{\varsigma}\left(\sum_{0 \leq i \leq m, 0<j<m} \omega(i, j) \tau^{i+j}\right)(\varsigma-\tau)^{\mu-1} d \tau \\
= & (1-\mu) \sum_{0 \leq i \leq m, 0<j<m} \omega(i, j) \varsigma^{i} \tau^{j}
\end{aligned}
$$




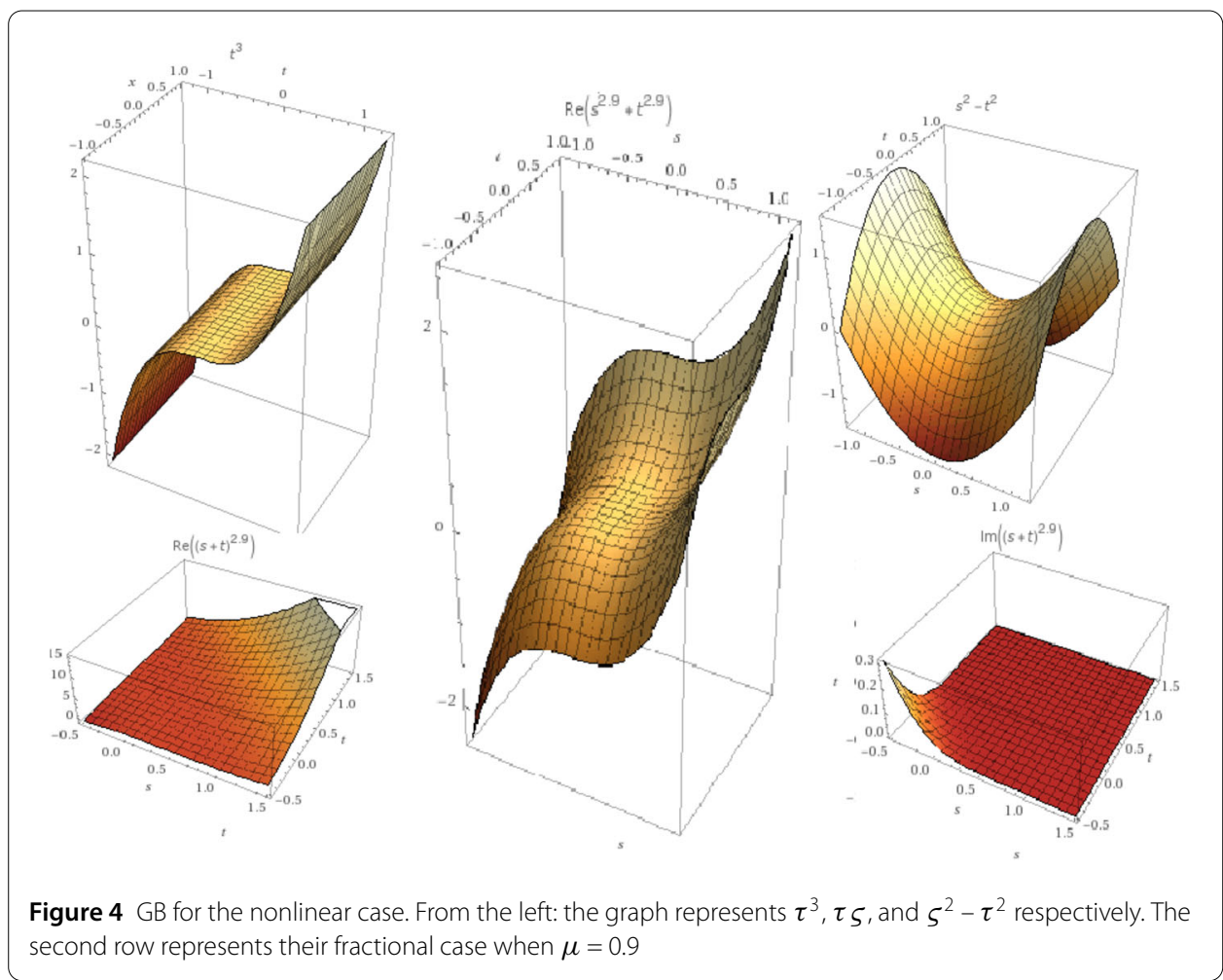

$$
\begin{aligned}
& +\frac{\mu}{\Gamma(\mu)} \sum_{0 \leq i \leq m, 0<j<m} \omega(i, j)\left(\int_{0}^{\varsigma} \tau^{i+j}(\varsigma-\tau)^{\mu-1} d \tau\right) \\
= & (1-\mu) \sum_{0 \leq i \leq m, 0<j<m} \omega(i, j) \varsigma^{i} \tau^{j}+\sum_{0 \leq i \leq m, 0<j<m} \omega(i, j)\left(J^{\mu} \varsigma^{i+j}\right) \\
= & (1-\mu) \sum_{0 \leq i \leq m, 0<j<m} \omega(i, j) \varsigma^{i} \tau^{j} \\
& +\sum_{0 \leq i \leq m, 0<j<m} \omega(i, j)\left((1-\mu) \varsigma^{i+j}+\frac{\mu \Gamma(i+j+1)}{\Gamma(i+j+1+\mu)} \varsigma^{i+j+\mu}\right) \\
= & \sum_{0 \leq i \leq m, 0<j<m} \omega(i, j)\left((1-\mu) \varsigma^{i} \tau^{j}+(1-\mu) \varsigma^{i+j}+\frac{\mu \Gamma(i+j+1)}{\Gamma(i+j+1+\mu)} \varsigma^{i+j+\mu}\right) .
\end{aligned}
$$

To determine the upper bound (approximated value) of $\omega(i, j)$, based on Theorem 2.2, we shall consider that these weights satisfy the upper bound of $\rho$, which is given by the formula

$$
\begin{aligned}
\omega(i, j) & =\frac{\Gamma(\mu)}{\Gamma(\mu)+(1-\mu)+\bar{\epsilon}_{\mu}} \\
& =\frac{\Gamma(\mu)}{\Gamma(\mu)+(2-\mu)+\frac{(\varsigma-\tau)^{\mu}}{\Gamma(\mu)}} .
\end{aligned}
$$

Note that $\lim _{\mu \rightarrow 1} \omega(\varsigma, \tau)=0.33$ providing that $\varsigma-\tau=1$. This value is approximated with the upper bound of $\rho$ in Example 3.2. By suggesting the solution of system (3) in terms of 


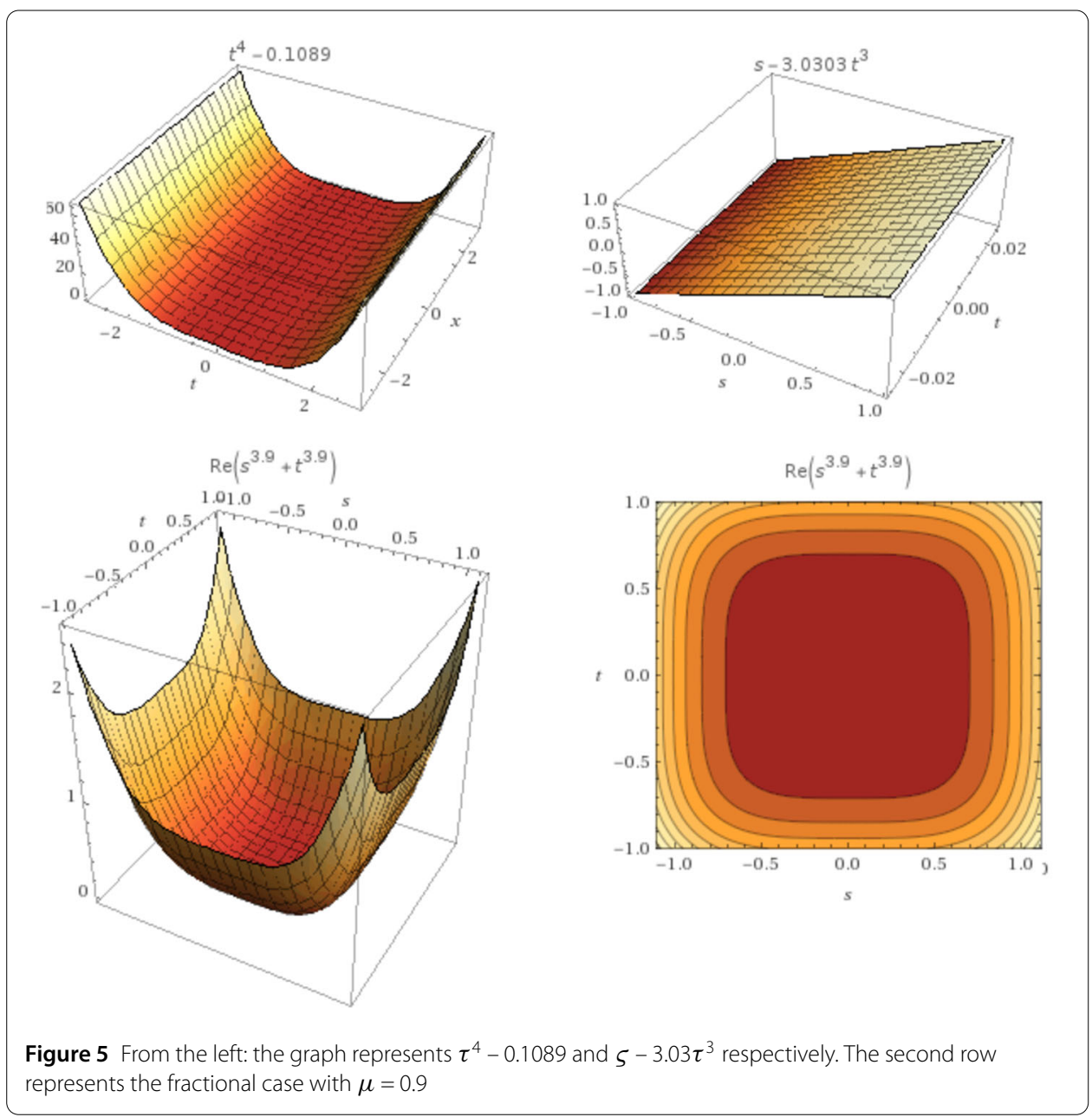

fractional Tutte polynomials, we have

$$
\chi(\varsigma)=\sum_{n=0}^{N} A_{n}(\varsigma, \tau) T_{n}^{\mu}(\varsigma, \tau), \quad \Upsilon(\varsigma)=\sum_{n=0}^{N} B_{n}(\varsigma, \tau) T_{n}^{\mu}(\varsigma, \tau) .
$$

We suppose that $\chi$ and $\Upsilon$ have the same gathering of roots as the original polynomials. That is, these polynomials can be recognized in a Grobner basis (GB). For linear functions in any number of variables, GB is analogous to Gaussian elimination. For example, if $(\chi(\varsigma), \Upsilon(\varsigma))=(\varsigma, \tau)$, then $\operatorname{GB}(\varsigma, \tau)=\{\varsigma, \tau\}$ (see Fig. 3). For nonlinear cases, we have the following results.

Example 3.4 $\mathrm{GB}(\chi(\varsigma), \Upsilon(\varsigma))=\mathrm{GB}\left(\tau^{2}-\varsigma^{2}, \tau * \varsigma\right)=\left\{\tau^{3}, \tau \varsigma, \varsigma^{2}-\tau^{2}\right\}$ with $(0,0)$ root $($ see Fig. 4).

Example $3.5 \mathrm{~GB}(\chi(\varsigma), \Upsilon(\varsigma))=\mathrm{GB}\left(\tau^{2}-\varsigma^{2}, \tau * \varsigma-0.33\right)=\left\{\tau^{4}-0.1089, \varsigma-3.03 \tau^{3}\right\}$ with the real root $\left(\frac{\sqrt{33}}{10}, \frac{\sqrt{33}}{10}\right)$ (see Fig. 5).

Example 3.6 $\mathrm{GB}(\chi(\varsigma), \Upsilon(\varsigma))=\mathrm{GB}\left(\varsigma^{3}-\tau^{2}, \varsigma-\tau\right)=\left\{\tau^{3}-\tau^{2}, \varsigma-\tau\right\}$ with two real roots $(0,0)$ and $(1,1)$ (see Fig. 6). 

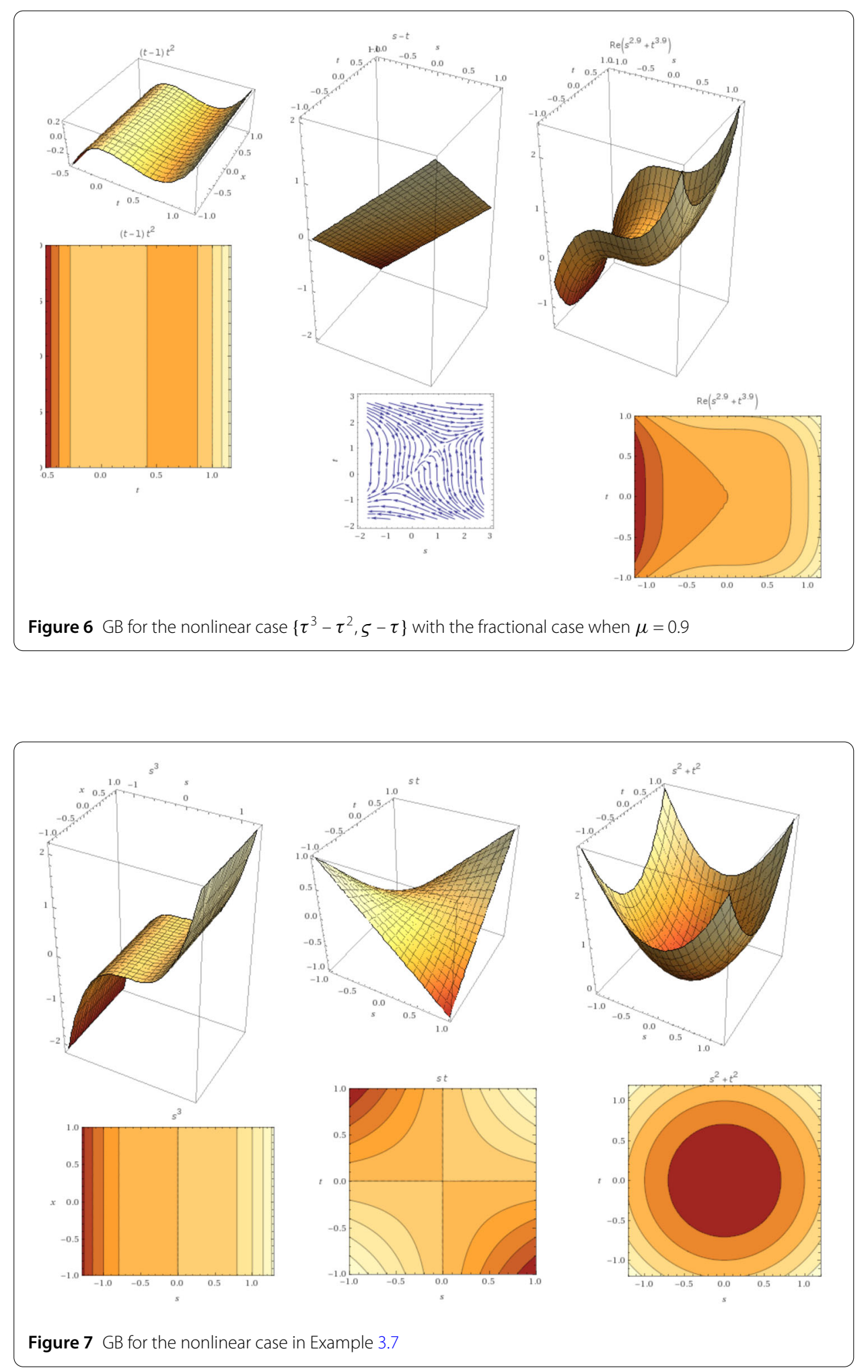

Example 3.7 $\mathrm{GB}(\chi(\varsigma), \Upsilon(\varsigma))=\mathrm{GB}\left(\varsigma^{3}+\tau^{2}, \varsigma * \tau\right)=\left\{\varsigma^{3}, \varsigma \tau, \varsigma^{2}+\tau^{2}\right\}$ with one real root $(0,0)$ (see Fig. 7). 

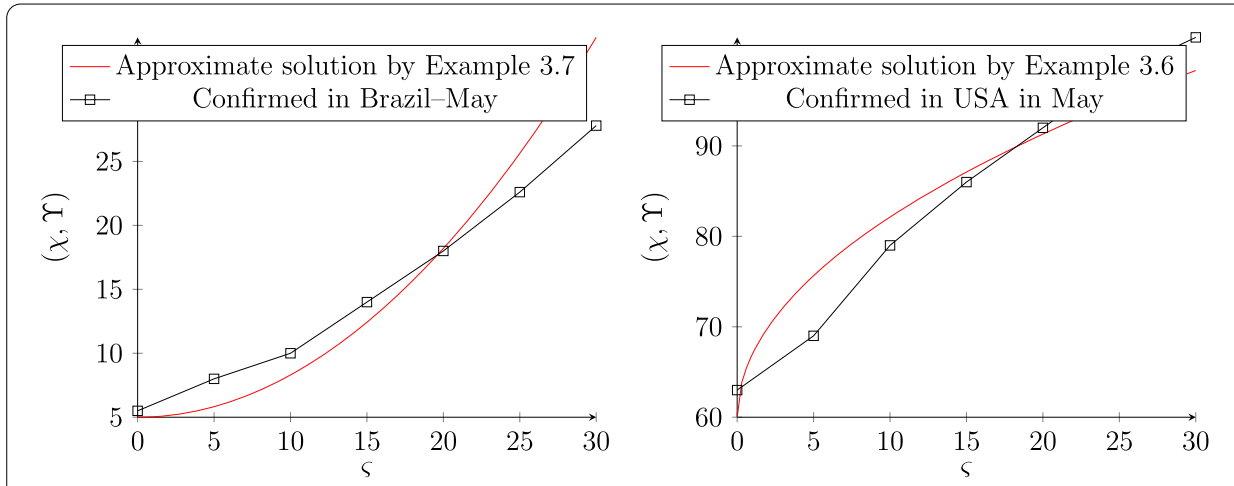

Figure 8 Approximate solution by using GB in May, where the data was given by $K=1000$. For Brazil, we select $\tau^{2}=5$, while for USA is $\tau=60$. The fractional order is $\mu=0.95$ and $\mu=0.98$ respectively.

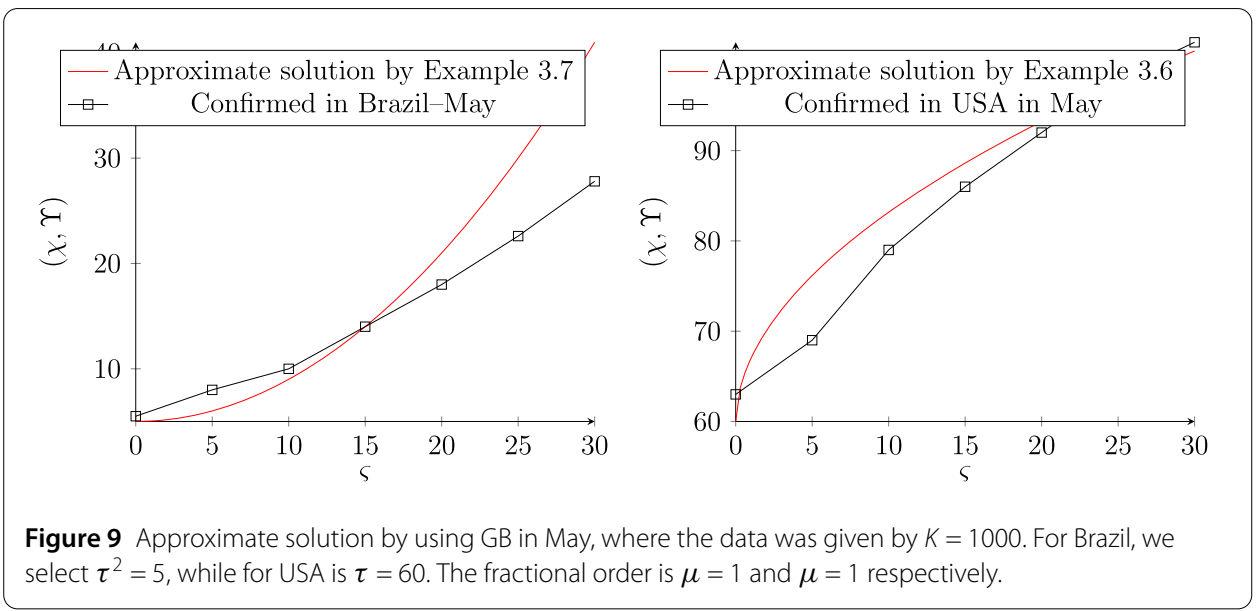

\subsection{Application}

In this subsection, we shall utilize live data regarding COVID-19, which were recorded in May. Figures 8 and 9 indicate live data which were recognized in May for Brazil and USA. By using the approximated solution, we see that the data converge to the result of different BG values where the value of $\tau$ indicates the starting situation. Experimentally, we indicate that the good result appears, when the value of fractional order $\mu \rightarrow 1$ and the interval of convergence solution is $\mu \in(0.8,0.99$ ].

\section{Conclusion}

By using the fractional calculus, type $\mathrm{ABC}$, we have generalized WCS. We have got two kinds of solutions, the first one is the optimal solution (see Theorem 3.1) using the concept of simulation function and the second is approximated solution using the GB set of polynomials of two variables $(\tau, \varsigma)$. The optimal solution brings the stability, oscillation, and periodicity. The second solution is validated for discrete data. In this investigation, we generalized the most popular graph polynomials called the Tutte polynomial and a variety of carefully related graph polynomials such as the harmonic, movement, reliability, and shelling polynomials. We also used the Tutte polynomial to demonstrate how graph polynomials may be both dedicated and generalized, and how they can convert information relevant to medical applications. We concluded with a brief conversation of compu- 
tational complexity deliberations. Different studies are presented using fractional calculus together with statistics and polynomials categories, which can be located in [19-25].

\section{Acknowledgements}

The authors would like to express their full thanks to the respected editor and reviewers for the deep advise, which improved our paper.

\section{Funding}

This research received no external funding.

\section{Availability of data and materials}

Data sharing not applicable to this article as no data-sets were generated or analyzed during the current study.

\section{Competing interests}

The authors declare that they have no competing interests.

\section{Authors' contributions}

All authors contributed equally and significantly to writing this article. All authors read and agreed to the published version of the manuscript.

\section{Author details}

${ }^{1}$ Nonlinear Dynamics Research Center (NDRC), Ajman University, Ajman, UAE. ${ }^{2}$ Department of Mathematics and Sciences, College of Humanities and Sciences, Ajman University, 346 Ajman, UAE. ${ }^{3}$ IEEE, 94086547, Kuala Lumpur, 59200, Malaysia.

\section{Publisher's Note}

Springer Nature remains neutral with regard to jurisdictional claims in published maps and institutional affiliations.

Received: 23 June 2021 Accepted: 7 July 2021 Published online: 29 July 2021

\section{References}

1. Wilson, H.R., Cowan, J.D.: A mathematical theory of the functional dynamics of cortical and thalamic nervous tissue. Kybernetik 13(2), 55-80 (1973)

2. Wilson, H.R., Blake, R., Lee, S.-H.: Dynamics of travelling waves in visual perception. Nature 412(6850), 907-910 (2001)

3. World, Organization, H.: Coronavirus disease (COVID-19) outbreak. https://www.who.int/emergencies/diseases/novel-coronavirus-2019

4. World Health Organization: Coronavirus disease (COVID-19) report. https://www.who.int/docs/default-source/coronaviruse/who-china-joint-mission-on-covid-19-final-report.pdf

5. Lanteri, D., Carco, D., Castorina, P.: How macroscopic laws describe complex dynamics: asymptomatic population and COVID-19 spreading (2020) arXiv:2003.12457

6. Atangana, A.: Modelling the spread of COVID-19 with new fractal-fractional operators: can the lockdown save mankind before vaccination? Chaos Solitons Fractals 136, 109860 (2020)

7. Atangana, A., Araz, S.: Modeling and forecasting the spread of COVID-19 with stochastic and deterministic approaches: Africa and Europe. Adv. Differ. Equ. 2021(1), 1 (2021)

8. Atangana, A., Araz, S.I: Mathematical model of COVID-19 spread in Turkey and South Africa: theory, methods, and applications. Adv. Differ. Equ. 2020(1), 1 (2020)

9. Musa, S.S., Qureshi, S., Zhao, S., Yusuf, A., Mustapha, U.T., He, D.: Mathematical modeling of COVID-19 epidemic with effect of awareness programs. Infect. Dis. Model. 6, 448-460 (2021)

10. Atangana, A.: A novel Covid-19 model with fractional differential operators with singular and non-singular kernels: analysis and numerical scheme based on Newton polynomial. Alex. Eng. J. 60(4), 3781-3806 (2021)

11. Memon, Z., Qureshi, S., Rasool Memon, B.: Assessing the role of quarantine and isolation as control strategies for COVID-19 outbreak: a case study. Chaos Solitons Fractals 144, 110655 (2021)

12. Caputo, M., Fabrizio, M.: A new definition of fractional derivative without singular kernel. Prog. Fract. Differ. Appl. 1(2), $1-13(2015)$

13. Atangana, A., Baleanu, D.: New fractional derivatives with nonlocal and non-singular kernel: theory and application to heat transfer model (2016) arXiv:1602.03408

14. Ahmad, Z., Arif, M., Ali, F., Khan, I., Nisar, K.S.: A report on COVID-19 epidemic in Pakistan using SEIR fractional model. Sci. Rep. 10(1), 1-14 (2020)

15. Khan, A., Alshehri, H.M., Abdeljawad, T., Al-Mdallal, Q.M., Khan, H.: Stability analysis of fractional nabla difference COVID-19 model. Results Phys. 22, 103888 (2021)

16. Abdo, M.S., Shah, K., Wahash, H.A., Panchal, S.K.: On a comprehensive model of the novel coronavirus (COVID-19) under Mittag-Leffler derivative. Chaos Solitons Fractals 135, 109867 (2020)

17. Patle, P.R., Patel, D.K., Arab, R.: Darbo type best proximity point results via simulation function with application. Afr. Math. 31, 833-845 (2020)

18. Tutte, W.T.: A contribution to the theory of chromatic polynomials. Can. J. Math. 6, 80-91 (1954)

19. Hadid, S.B., Ibrahim, R.W., Altulea, D., Momani, S.: Solvability and stability of a fractional dynamical system of the growth of COVID-19 with approximate solution by fractional Chebyshev polynomials. Adv. Differ. Equ. 2020(1), 1 (2020)

20. Hasan, A.M., Al-Jawad, M.M., Jalab, H.A., Shaiba, H., Ibrahim, R.W., AL-Shamasneh, A.R.: Classification of COVID-19 coronavirus, pneumonia and healthy lungs in CT scans using q-deformed entropy and deep learning features. Entropy 22(5), 517 (2020) 
21. Ibrahim, R.W., Altulea, D., Elobaid, R.M.: Dynamical system of the growth of COVID-19 with controller. Adv. Differ. Equ. 2021(1), 1 (2021)

22. Momani, S., Ibrahim, R.W., Hadid, S.B.: Susceptible-infected-susceptible epidemic discrete dynamic system based on Tsallis entropy. Entropy 22(7), 769 (2020)

23. Irfan, O., Muttalib, F., Tang, K., Jiang, L., Lassi, Z.S., Bhutta, Z.: Clinical characteristics, treatment and outcomes of paediatric COVID-19: a systematic review and meta-analysis. Arch. Dis. Child. 106(5), 440-448 (2021)

24. Alderremy, A.A., Gómez-Aguilar, J.F., Aly, S., Saad, K.M.: A fuzzy fractional model of coronavirus (COVID-19) and its study with Legendre spectral method. Results Phys. 21, 103773 (2021)

25. Yousaf, M., Zahir, S., Riaz, M., Hussain, S.M., Shah, K.: Statistical analysis of forecasting COVID-19 for upcoming month in Pakistan. Chaos Solitons Fractals 138, 109926 (2020)

Submit your manuscript to a SpringerOpen ${ }^{\circ}$ journal and benefit from:

- Convenient online submission

- Rigorous peer review

- Open access: articles freely available online

- High visibility within the field

- Retaining the copyright to your article

Submit your next manuscript at $\gg$ springeropen.com 